Supplement of

\title{
A photogrammetry-based approach for soil bulk density measurements with an emphasis on applications to cosmogenic nuclide analysis
}

Joel Mohren et al.

Correspondence to: Joel Mohren (joel.mohren@uni-koeln.de)

The copyright of individual parts of the supplement might differ from the CC BY 4.0 License. 
S1 Flower pot design and picturing scenarios

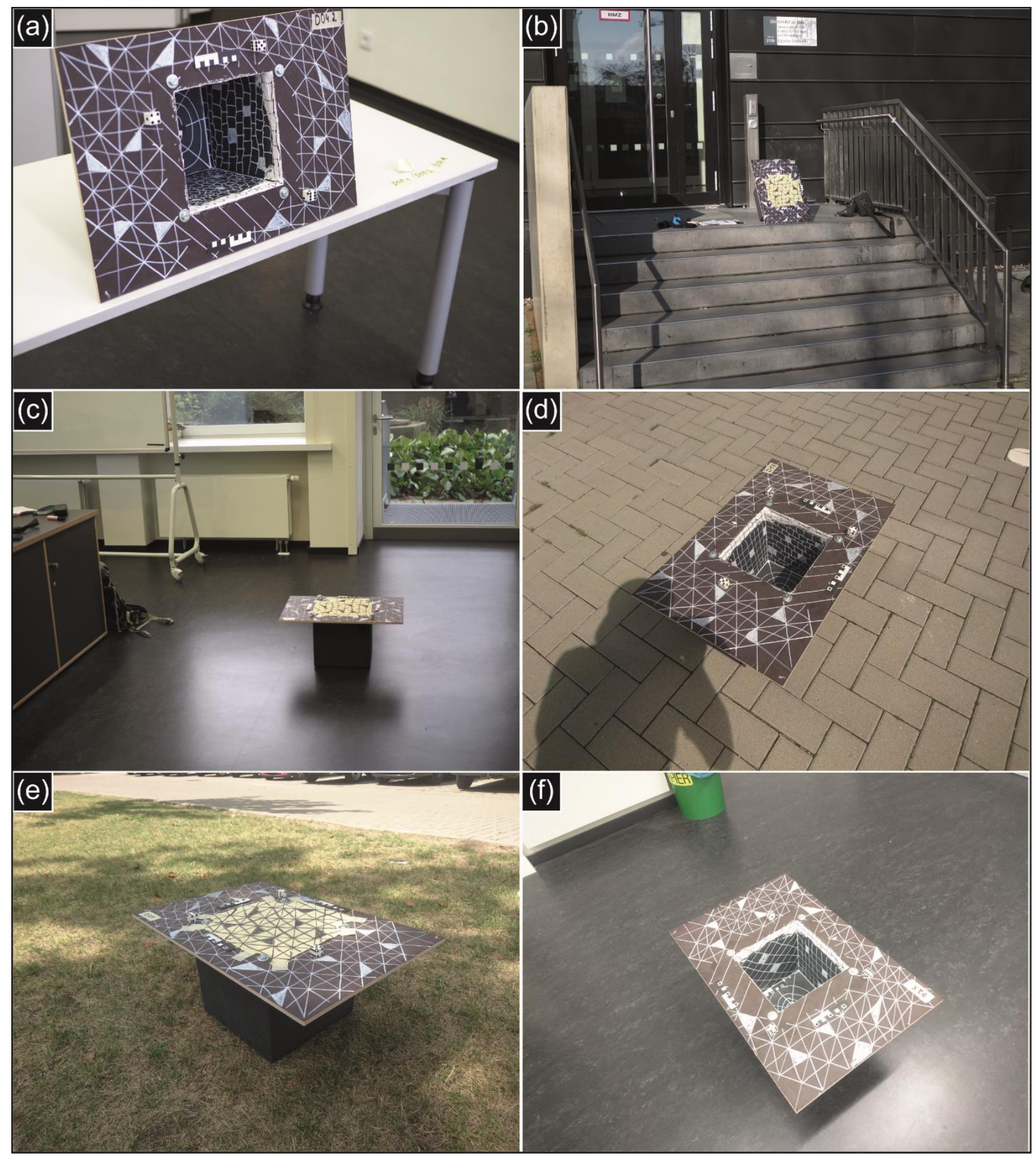

Figure S1. Flower pot design and examples for different settings: (a) D01 - indoors, natural light dominant (tilted); (b) D11 - outdoors, direct sunlight (tilted); (c) D16 - indoors, natural light dominant; (d) D17 outdoors, direct sunlight; (e) D20 - outdoors, no direct sunlight; $(f)$ D25 - indoors, artificial light dominant. A black/dark brown and white pattern was painted on the initially dark surfaces of the flower pot and the board in order to enhance image textures. The flower pot could be sealed by a thin fabric in order to simulate the pre-dug surface (D11, D16, D20). 


\section{S2 BD17 field tests}

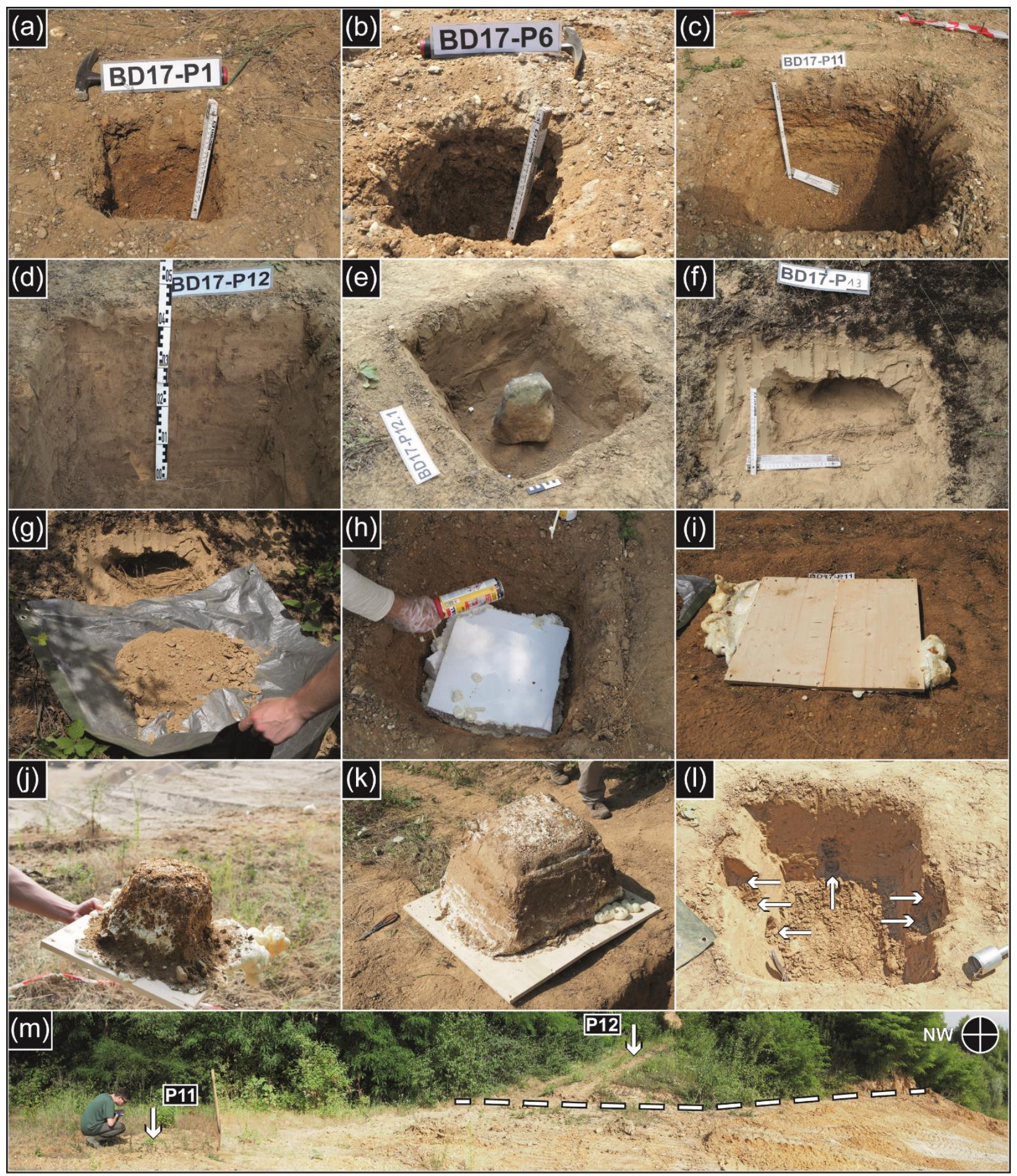

Figure S2. Pictures taken during the field tests in the gravel pit near Cologne, Germany. Different soil pits [(a) - BD17-P01; (b) - BD17-P06; (c) - BD17-P11; (d) - BD17-P12; (e) - BD17-P12.1] and a cavity [(f) - BD17-P13] were dug into gravel and loess layers within a radius of $\sim 50 \mathrm{~m}$. The excavated material was collected on a tarp $(g)$ and weighed afterwards using a digital spring balance (luggage scale). The pits were filled with polyurethane foam and polystyrene blocks in order to obtain casts reflecting the volume of the respective pit after cooling $(h-k)$. Small cores were taken from some sites, including BD17-P12 [(l); 
white arrows indicate sampling locations]. Excavation work in the gravel pit has exposed the Pleistocene gravels which are overlain by loess deposits [( $\mathrm{m})$; dashed line indicates the contact between loess (above) and gravel layers (below)].

\section{S3 Altos de Talinay TCN sampling campaign}

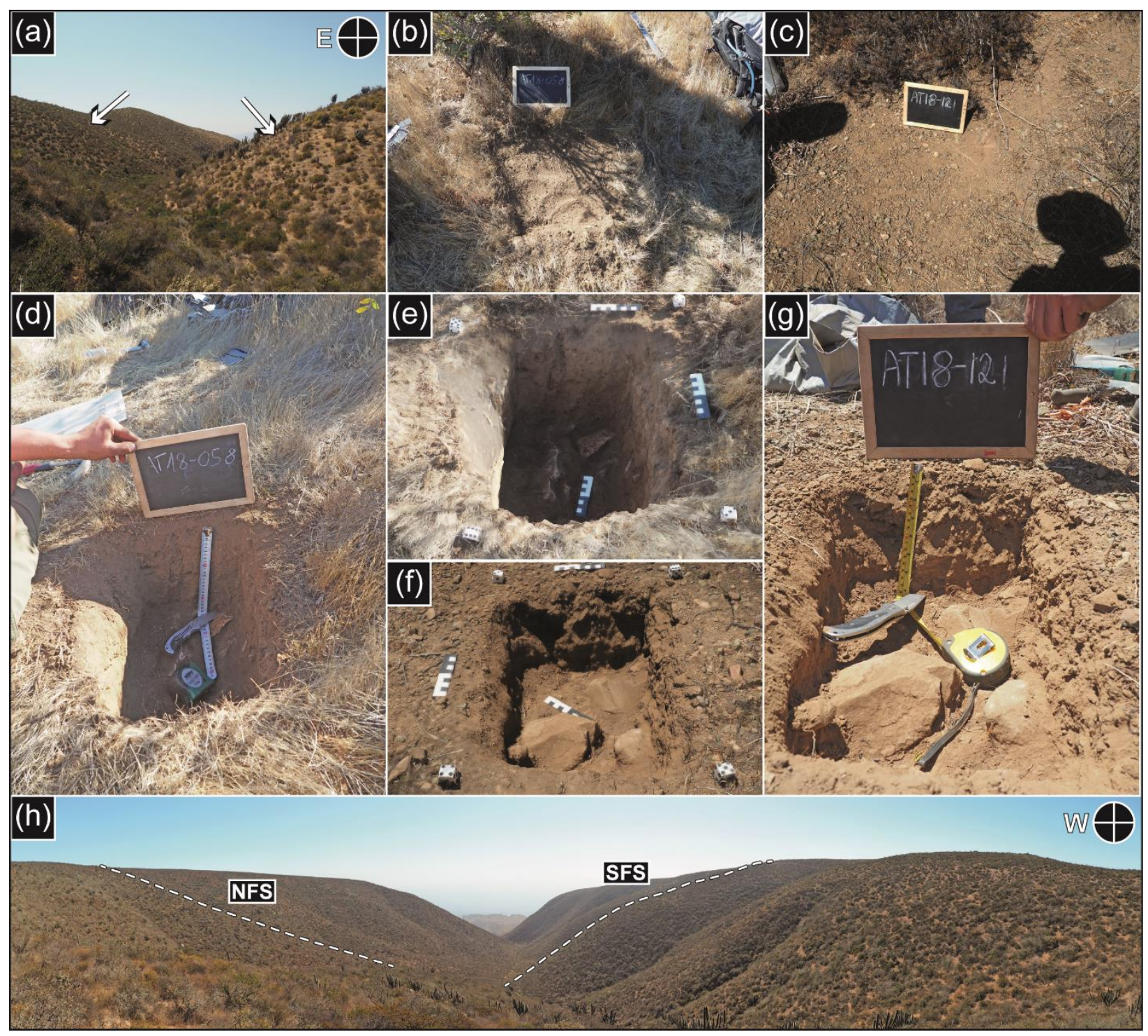

Figure S3. Study area characteristics and examples for sampling sites in the Altos de Talinay, northern central Chile. In the E-W oriented catchments, strong contrasts in vegetation cover and slope morphology between SFS and NFS can be observed $(a, h)$. Before sampling for TCN below the regolith/saprolite or bedrock boundary, pictures were taken from the pre-dug surface [SFS - (b); NFS - (c)]. After sampling, the procedure was repeated by acquiring photographs from the sampling pits [SFS - (d); NFS - $(g)]$. The image datasets were then used to model the pits [(SFS-(e); NFS-(f)]. 
Table S1. Sample details and calculated field-state bulk densities of the AT17/18 sampling campaign.

\begin{tabular}{|c|c|c|c|c|c|c|c|c|c|c|}
\hline Sample ID & $\begin{array}{r}\text { Loca } \\
\left({ }^{\circ}\right.\end{array}$ & & $\begin{array}{l}\text { Elevation } \\
(\mathrm{m} \text { a.s.l. })^{\mathrm{a}}\end{array}$ & Catchment & Exposition & $\begin{array}{c}\mathrm{z} \\
(\mathrm{cm})^{\mathrm{b}}\end{array}$ & $\begin{array}{l}m_{e, f} \\
(g)^{c}\end{array}$ & $\begin{array}{c}\text { Number } \\
\text { of } \\
\text { pictures }\end{array}$ & $\begin{array}{l}\mathrm{V}_{\mathrm{SfM}} \\
\left(\mathrm{cm}^{3}\right)^{\mathrm{d}}\end{array}$ & $\begin{array}{c}\rho_{\mathrm{B}, \mathrm{f}} \\
\left(\mathrm{g} \mathrm{cm}^{-3}\right)^{\mathrm{e}}\end{array}$ \\
\hline AT17-001(1) & -30.4613 & -71.6522 & 338 & $\mathrm{C} 1$ & Ridgetop & 22 & $11150 \pm 71$ & 35 & $8559 \pm 70$ & $1.30 \pm 0.01$ \\
\hline AT17-002 & -30.4623 & -71.6546 & 325 & $\mathrm{C} 1$ & Ridgetop & 19 & $10500 \pm 71$ & 35 & $7327 \pm 70$ & $1.43 \pm 0.02$ \\
\hline AT17-023 & -30.4610 & -71.6486 & 368 & $\mathrm{C} 1$ & South & 10 & $5000 \pm 71$ & 27 & $3630 \pm 70$ & $1.38 \pm 0.03$ \\
\hline AT17-024 & -30.4616 & -71.6487 & 359 & $\mathrm{C} 1$ & South & 6 & $6150 \pm 71$ & 45 & $4143 \pm 70$ & $1.48 \pm 0.03$ \\
\hline AT17-025 & -30.4622 & -71.6487 & 344 & $\mathrm{C} 1$ & South & 23 & $10800 \pm 71$ & 34 & $7574 \pm 70$ & $1.43 \pm 0.02$ \\
\hline AT17-026 & -30.4627 & -71.6489 & 323 & $\mathrm{C} 1$ & South & 28 & $33250 \pm 71$ & 43 & $36463 \pm 70$ & $0.91 \pm 0.00$ \\
\hline AT17-027 & -30.4632 & -71.6492 & 296 & $\mathrm{C} 1$ & South & 22 & $44500 \pm 100$ & 91 & $30164 \pm 70$ & $1.48 \pm 0.00$ \\
\hline AT17-028(1) & -30.4660 & -71.6487 & 387 & $\mathrm{C} 1$ & Ridgetop & 5 & $10950 \pm 71$ & 42 & $8562 \pm 70$ & $1.28 \pm 0.01$ \\
\hline AT17-029(1) & -30.4658 & -71.6499 & 367 & $\mathrm{C} 1$ & North & 8 & $8650 \pm 71$ & 80 & $6940 \pm 70$ & $1.25 \pm 0.02$ \\
\hline AT17-030 & -30.4653 & -71.6498 & 360 & $\mathrm{C} 1$ & North & 9 & $9100 \pm 71$ & 70 & $6740 \pm 70$ & $1.35 \pm 0.02$ \\
\hline AT17-031 & -30.4647 & -71.6498 & 344 & $\mathrm{C} 1$ & North & 6 & $9750 \pm 71$ & 75 & $6377 \pm 70$ & $1.53 \pm 0.02$ \\
\hline AT17-032TS & -30.4643 & -71.6502 & 324 & $\mathrm{C} 1$ & North & 5 & $3400 \pm 71$ & 59 & $2028 \pm 70$ & $1.68 \pm 0.07$ \\
\hline AT17-033 & -30.4638 & -71.6499 & 304 & $\mathrm{C} 1$ & North & 2 & $2800 \pm 71$ & 36 & $1721 \pm 70$ & $1.63 \pm 0.08$ \\
\hline AT18-034 & -30.4663 & -71.6092 & 486 & $\mathrm{C} 2$ & North & 10 & $25050 \pm 100$ & 29 & $15654 \pm 70$ & $1.60 \pm 0.01$ \\
\hline AT18-036 & -30.4661 & -71.6093 & 480 & $\mathrm{C} 2$ & North & 9 & $9550 \pm 71$ & 27 & $6279 \pm 70$ & $1.52 \pm 0.02$ \\
\hline AT18-037 & -30.4658 & -71.6093 & 463 & $\mathrm{C} 2$ & North & 15 & $19550 \pm 71$ & 32 & $13064 \pm 70$ & $1.50 \pm 0.01$ \\
\hline AT18-038 & -30.4655 & -71.6093 & 451 & $\mathrm{C} 2$ & North & 24 & $26700 \pm 100$ & 35 & $15255 \pm 70$ & $1.75 \pm 0.01$ \\
\hline AT18-039 & -30.4652 & -71.6093 & 435 & $\mathrm{C} 2$ & North & 21 & $17850 \pm 71$ & 31 & $11373 \pm 70$ & $1.57 \pm 0.01$ \\
\hline AT18-040 & -30.4653 & -71.6093 & 440 & $\mathrm{C} 2$ & North & 30 & $28550 \pm 100$ & 34 & $17107 \pm 70$ & $1.67 \pm 0.01$ \\
\hline AT18-041 & -30.4650 & -71.6093 & 426 & $\mathrm{C} 2$ & North & 14 & $9350 \pm 71$ & 46 & $5662 \pm 70$ & $1.65 \pm 0.02$ \\
\hline AT18-042 & -30.4647 & -71.6092 & 409 & $\mathrm{C} 2$ & North & 30 & $29700 \pm 100$ & 39 & $17060 \pm 70$ & $1.74 \pm 0.01$ \\
\hline AT18-044 & -30.4642 & -71.6090 & 383 & $\mathrm{C} 2$ & North & 23 & $25600 \pm 100$ & 58 & $18579 \pm 70$ & $1.38 \pm 0.01$ \\
\hline AT18-051 & -30.4639 & -71.6090 & 371 & $\mathrm{C} 2$ & North & 29 & $29650 \pm 71$ & 42 & $15525 \pm 70$ & $1.91 \pm 0.01$ \\
\hline AT18-052 & -30.4637 & -71.6089 & 358 & $\mathrm{C} 2$ & North & 16 & $18750 \pm 71$ & 42 & $11340 \pm 70$ & $1.65 \pm 0.01$ \\
\hline AT18-053 & -30.4634 & -71.6098 & 375 & $\mathrm{C} 2$ & South & 24 & $53400 \pm 141$ & 55 & $34590 \pm 70$ & $1.54 \pm 0.01$ \\
\hline AT18-054 & -30.4636 & -71.6098 & 366 & $\mathrm{C} 2$ & South & 23 & $33400 \pm 122$ & 44 & $23699 \pm 70$ & $1.41 \pm 0.01$ \\
\hline AT18-055 & -30.4631 & -71.6100 & 404 & $\mathrm{C} 2$ & South & 13 & $21500 \pm 71$ & 53 & $14437 \pm 70$ & $1.49 \pm 0.01$ \\
\hline AT18-056 & -30.4628 & -71.6103 & 421 & $\mathrm{C} 2$ & South & 25 & $43500 \pm 122$ & 37 & $35659 \pm 70$ & $1.22 \pm 0.00$ \\
\hline AT18-057 & -30.4625 & -71.6105 & 439 & $\mathrm{C} 2$ & South & 30 & $40500 \pm 71$ & 40 & $24884 \pm 70$ & $1.63 \pm 0.01$ \\
\hline AT18-058 & -30.4622 & -71.6107 & 455 & $\mathrm{C} 2$ & South & 24 & $42600 \pm 122$ & 34 & $28733 \pm 70$ & $1.48 \pm 0.01$ \\
\hline AT18-059 & -30.4632 & -71.6099 & 392 & $\mathrm{C} 2$ & South & 23 & $26950 \pm 100$ & 42 & $18845 \pm 70$ & $1.43 \pm 0.01$ \\
\hline AT18-060 & -30.4621 & -71.6110 & 462 & $\mathrm{C} 2$ & South & 31 & $33850 \pm 100$ & 70 & $25213 \pm 70$ & $1.34 \pm 0.01$ \\
\hline AT18-061 & -30.4620 & -71.6113 & 468 & $\mathrm{C} 2$ & South & 20 & $30200 \pm 100$ & 38 & $18356 \pm 70$ & $1.65 \pm 0.01$ \\
\hline AT18-062 & -30.4618 & -71.6115 & 470 & $\mathrm{C} 2$ & South & 20 & $26900 \pm 71$ & 43 & $17678 \pm 70$ & $1.52 \pm 0.01$ \\
\hline AT18-063 & -30.4609 & -71.6131 & 492 & $\mathrm{C} 2$ & Ridgetop & 5 & $10450 \pm 71$ & 42 & $7490 \pm 70$ & $1.40 \pm 0.02$ \\
\hline AT18-102(2) & -30.3922 & -71.6474 & 428 & $\mathrm{C} 5$ & Ridgetop & 3 & $2150 \pm 71$ & 35 & $2356 \pm 70$ & $0.91 \pm 0.04$ \\
\hline AT18-106 & -30.3862 & -71.6487 & 455 & C5 & Ridgetop & 22 & $29600 \pm 100$ & 57 & $20437 \pm 70$ & $1.45 \pm 0.01$ \\
\hline AT18-108 & -30.3881 & -71.6500 & 447 & $\mathrm{C} 5$ & South & 8 & $9509 \pm 71$ & 38 & $5757 \pm 70$ & $1.65 \pm 0.02$ \\
\hline AT18-109 & -30.3887 & -71.6502 & 442 & $\mathrm{C} 5$ & South & 15 & $27950 \pm 71$ & 42 & $\mid 16597 \pm 70$ & $1.68 \pm 0.01$ \\
\hline
\end{tabular}




\begin{tabular}{|c|c|c|c|c|c|c|c|c|c|c|}
\hline AT18-111 & -30.3890 & -71.6502 & 436 & $\mathrm{C} 5$ & South & 10 & $8200 \pm 71$ & 36 & $5186 \pm 70$ & $1.58 \pm 0.03$ \\
\hline AT18-112 & -30.3895 & -71.6504 & 424 & $\mathrm{C} 5$ & South & 12 & $10750 \pm 71$ & 78 & $8015 \pm 70$ & $1.34 \pm 0.01$ \\
\hline AT18-113 & -30.3897 & -71.6505 & 415 & $\mathrm{C} 5$ & South & 10 & $8950 \pm 71$ & 42 & $5840 \pm 70$ & $1.53 \pm 0.02$ \\
\hline AT18-114 & -30.3899 & -71.6506 & 405 & $\mathrm{C} 5$ & South & 11 & $15350 \pm 71$ & 38 & $11033 \pm 70$ & $1.39 \pm 0.01$ \\
\hline AT18-115 & -30.3902 & -71.6508 & 395 & $\mathrm{C} 5$ & South & 16 & $28850 \pm 71$ & 72 & $20737 \pm 70$ & $1.39 \pm 0.01$ \\
\hline AT18-116 & -30.3905 & -71.6508 & 378 & $\mathrm{C} 5$ & South & 13 & $16900 \pm 71$ & 48 & $13345 \pm 70$ & $1.27 \pm 0.01$ \\
\hline AT18-117 & -30.3908 & -71.6509 & 358 & $\mathrm{C} 5$ & South & 19 & $9650 \pm 71$ & 87 & $8757 \pm 70$ & $1.10 \pm 0.01$ \\
\hline AT18-120 & -30.3913 & -71.6510 & 319 & $\mathrm{C} 5$ & South & 42 & $33750 \pm 122$ & 38 & $25663 \pm 70$ & $1.32 \pm 0.01$ \\
\hline AT18-121 & -30.3920 & -71.6512 & 321 & $\mathrm{C} 5$ & North & 17 & $22750 \pm 71$ & 57 & $12644 \pm 70$ & $1.80 \pm 0.01$ \\
\hline AT18-122 & -30.3923 & -71.6510 & 334 & $\mathrm{C} 5$ & North & 13 & $16850 \pm 71$ & 41 & $9757 \pm 70$ & $1.73 \pm 0.01$ \\
\hline AT18-123 & -30.3926 & -71.6509 & 348 & $\mathrm{C} 5$ & North & 21 & $24200 \pm 100$ & 39 & $17393 \pm 70$ & $1.39 \pm 0.01$ \\
\hline AT18-124 & -30.3928 & -71.6508 & 363 & $\mathrm{C} 5$ & North & 18 & $19500 \pm 71$ & 43 & $13541 \pm 70$ & $1.44 \pm 0.01$ \\
\hline AT18-127 & -30.3935 & -71.6504 & 397 & $\mathrm{C} 5$ & North & 16 & $20400 \pm 71$ & 77 & $15386 \pm 70$ & $1.33 \pm 0.01$ \\
\hline AT18-129 & -30.3937 & -71.6504 & 405 & $\mathrm{C} 5$ & North & 7 & $7700 \pm 71$ & 55 & $5881 \pm 70$ & $1.31 \pm 0.02$ \\
\hline AT18-132 & -30.3940 & -71.6504 & 416 & $\mathrm{C} 5$ & North & 13 & $11650 \pm 71$ & 76 & $7639 \pm 70$ & $1.53 \pm 0.02$ \\
\hline AT18-133 & -30.3943 & -71.6504 & 429 & $\mathrm{C} 5$ & North & 7 & $5650 \pm 71$ & 56 & $4329 \pm 70$ & $1.31 \pm 0.03$ \\
\hline AT18-136 & -30.3958 & -71.6498 & 478 & $\mathrm{C} 5$ & North & 10 & $9350 \pm 71$ & 38 & $5429 \pm 70$ & $1.72 \pm 0.03$ \\
\hline AT18-137 & -30.3950 & -71.6505 & 457 & $\mathrm{C} 5$ & North & 2 & $1550 \pm 71$ & 64 & $1285 \pm 70$ & $1.21 \pm 0.09$ \\
\hline AT18-138 & -30.4001 & -71.6510 & 524 & $\mathrm{C} 5$ & Ridgetop & 4 & $4850 \pm 71$ & 62 & $3489 \pm 70$ & $1.39 \pm 0.03$ \\
\hline AT18-139 & -30.3948 & -71.6504 & 448 & $\mathrm{C} 5$ & North & 8 & $8800 \pm 71$ & 73 & $6319 \pm 70$ & $1.39 \pm 0.02$ \\
\hline AT18-145 & -30.4613 & -71.6487 & 363 & $\mathrm{C} 1$ & South & 20 & $17900 \pm 71$ & 50 & $13622 \pm 70$ & $1.31 \pm 0.01$ \\
\hline AT18-146 & -30.4619 & -71.6487 & 352 & $\mathrm{C} 1$ & South & 21 & $12850 \pm 71$ & 54 & $8929 \pm 70$ & $1.44 \pm 0.01$ \\
\hline AT18-147 & -30.4624 & -71.6489 & 336 & $\mathrm{C} 1$ & South & 26 & $19050 \pm 71$ & 39 & $16122 \pm 70$ & $1.18 \pm 0.01$ \\
\hline AT18-148 & -30.4629 & -71.6491 & 310 & $\mathrm{C} 1$ & South & 14 & $5850 \pm 71$ & 43 & $5092 \pm 70$ & $1.15 \pm 0.02$ \\
\hline AT18-149 & -30.4635 & -71.6499 & 293 & $\mathrm{C} 1$ & North & 15 & $15700 \pm 71$ & 83 & $10571 \pm 70$ & $1.49 \pm 0.01$ \\
\hline AT18-150 & -30.4640 & -71.6500 & 313 & $\mathrm{C} 1$ & North & 8 & $5700 \pm 71$ & 79 & $3301 \pm 70$ & $1.73 \pm 0.04$ \\
\hline AT18-151 & -30.4645 & -71.6500 & 335 & $\mathrm{C} 1$ & North & 14 & $11350 \pm 71$ & 48 & $8909 \pm 70$ & $1.27 \pm 0.01$ \\
\hline AT18-152 & -30.4650 & -71.6499 & 353 & $\mathrm{C} 1$ & North & 5 & $6300 \pm 71$ & 82 & $3848 \pm 70$ & $1.64 \pm 0.04$ \\
\hline AT18-153 & -30.4655 & -71.6499 & 365 & $\mathrm{C} 1$ & North & 11 & $13650 \pm 71$ & 49 & $10023 \pm 70$ & $1.36 \pm 0.01$ \\
\hline AT18-154 & -30.4658 & -71.6495 & 373 & $\mathrm{C} 1$ & Ridgetop & 8 & $26400 \pm 71$ & 50 & $12482 \pm 70$ & $2.11 \pm 0.01$ \\
\hline
\end{tabular}

${ }^{a}$ DTM-derived.

${ }^{b}$ Soil pit depth below the surface as measured in the field.

${ }^{c}$ Field-state excavation mass, uncertainty equals the accuracy of the hand balance used.

${ }^{d}$ SfM-MVS photogrammetry-based pit volumes. Uncertainties are $1 \sigma$ as obtained from the tests performed on the artificial pit.

${ }^{e}$ Field-state bulk density. 
Table S2. Sample aliquot measurements and calculated dry densities.

\begin{tabular}{|c|c|c|c|c|c|c|c|c|c|c|}
\hline Sample ID & $\begin{array}{l}\text { Elevation } \\
(\mathrm{m} \text { a.s.l. })^{\mathrm{a}}\end{array}$ & Catchment & Exposition & $\begin{array}{c}\mathrm{Z} \\
(\mathrm{cm})^{\mathrm{b}}\end{array}$ & $\begin{array}{l}m_{a, f} \\
(g)^{c}\end{array}$ & $\begin{array}{l}m_{a, d} \\
(g)^{d}\end{array}$ & $\begin{array}{l}\mathrm{m}_{\mathrm{a}, \mathrm{r}} \\
(\mathrm{g})^{\mathrm{e}}\end{array}$ & $\begin{array}{c}\rho_{\mathrm{BR}} \\
\left(\mathrm{g} \mathrm{cm}^{-3}\right)^{\mathrm{f}}\end{array}$ & $\begin{array}{c}\rho_{\mathrm{B}, \mathrm{d}} \\
\left(\mathrm{g} \mathrm{cm}^{-3}\right)^{\mathrm{g}}\end{array}$ & $\begin{array}{c}\rho_{\mathrm{d}} \\
\left(\mathrm{g} \mathrm{cm}^{-3}\right)^{\mathrm{h}}\end{array}$ \\
\hline AT17-001(1) & 338 & $\mathrm{C} 1$ & Ridgetop & 22 & $364.1 \pm 0.1$ & $360.5 \pm 0.1$ & $46.2 \pm 0.1$ & $2.82 \pm 0.11$ & $1.29 \pm 0.01$ & $1.19 \pm 0.05$ \\
\hline AT17-002 & 325 & $\mathrm{C} 1$ & Ridgetop & 19 & $204.3 \pm 0.1$ & $202.9 \pm 0.1$ & $34.1 \pm 0.1$ & $2.82 \pm 0.11$ & $1.42 \pm 0.02$ & $1.29 \pm 0.05$ \\
\hline AT17-023 & 368 & $\mathrm{C} 1$ & South & 10 & $468.0 \pm 0.1$ & $463.2 \pm 0.1$ & $97.7 \pm 0.1$ & $2.82 \pm 0.11$ & $1.36 \pm 0.03$ & $1.20 \pm 0.06$ \\
\hline AT17-024 & 359 & $\mathrm{C} 1$ & South & 6 & $224.9 \pm 0.1$ & $222.3 \pm 0.1$ & $66.6 \pm 0.1$ & $2.82 \pm 0.11$ & $1.47 \pm 0.03$ & $1.22 \pm 0.06$ \\
\hline AT17-025 & 344 & $\mathrm{C} 1$ & South & 23 & $363.8 \pm 0.1$ & $358.8 \pm 0.1$ & $42.4 \pm 0.1$ & $2.82 \pm 0.11$ & $1.41 \pm 0.02$ & $1.32 \pm 0.05$ \\
\hline AT17-026 & 323 & $\mathrm{C} 1$ & South & 28 & $457.2 \pm 0.1$ & $446.3 \pm 0.1$ & $56.6 \pm 0.1$ & $2.82 \pm 0.11$ & $0.89 \pm 0.00$ & $0.81 \pm 0.03$ \\
\hline AT17-027 & 296 & $\mathrm{C} 1$ & South & 22 & $619.7 \pm 0.1$ & $612.0 \pm 0.1$ & $38.3 \pm 0.1$ & $2.82 \pm 0.11$ & $1.46 \pm 0.00$ & $1.41 \pm 0.05$ \\
\hline AT17-028(1) & 387 & $\mathrm{C} 1$ & Ridgetop & 5 & $106.7 \pm 0.1$ & $105.5 \pm 0.1$ & $10.7 \pm 0.1$ & $2.82 \pm 0.11$ & $1.26 \pm 0.01$ & $1.19 \pm 0.05$ \\
\hline AT17-029(1) & 367 & $\mathrm{C} 1$ & North & 8 & $121.6 \pm 0.1$ & $120.3 \pm 0.1$ & $7.7 \pm 0.1$ & $2.82 \pm 0.11$ & $1.23 \pm 0.02$ & $1.19 \pm 0.05$ \\
\hline AT17-030 & 360 & $\mathrm{C} 1$ & North & 9 & $203.3 \pm 0.1$ & $201.9 \pm 0.1$ & $42.5 \pm 0.1$ & $2.82 \pm 0.11$ & $1.34 \pm 0.02$ & $1.18 \pm 0.05$ \\
\hline AT17-031 & 344 & $\mathrm{C} 1$ & North & 6 & $291.4 \pm 0.1$ & $288.9 \pm 0.1$ & $51.9 \pm 0.1$ & $2.82 \pm 0.11$ & $1.52 \pm 0.02$ & $1.38 \pm 0.06$ \\
\hline AT17-033 & 304 & $\mathrm{C} 1$ & North & 2 & $216.4 \pm 0.1$ & $214.8 \pm 0.1$ & $76.7 \pm 0.1$ & $2.82 \pm 0.11$ & $1.62 \pm 0.08$ & $1.31 \pm 0.10$ \\
\hline AT18-034 & 486 & $\mathrm{C} 2$ & North & 10 & $432.9 \pm 0.1$ & $429.9 \pm 0.1$ & $35.2 \pm 0.1$ & $2.82 \pm 0.11$ & $1.59 \pm 0.01$ & $1.53 \pm 0.06$ \\
\hline AT18-037 & 463 & $\mathrm{C} 2$ & North & 15 & $335.3 \pm 0.1$ & $330.0 \pm 0.1$ & $74.0 \pm 0.1$ & $2.82 \pm 0.11$ & $1.47 \pm 0.01$ & $1.29 \pm 0.05$ \\
\hline AT18-038 & 451 & $\mathrm{C} 2$ & North & 24 & $809.7 \pm 0.1$ & $801.2 \pm 0.1$ & $282.9 \pm 0.1$ & $2.82 \pm 0.11$ & $1.73 \pm 0.01$ & $1.43 \pm 0.06$ \\
\hline AT18-041 & 426 & $\mathrm{C} 2$ & North & 14 & $384.2 \pm 0.1$ & $378.6 \pm 0.1$ & $94.0 \pm 0.1$ & $2.82 \pm 0.11$ & $1.63 \pm 0.02$ & $1.43 \pm 0.06$ \\
\hline AT18-051 & 371 & $\mathrm{C} 2$ & North & 29 & $236.9 \pm 0.1$ & $232.8 \pm 0.1$ & $65.9 \pm 0.1$ & $2.82 \pm 0.11$ & $1.88 \pm 0.01$ & $1.66 \pm 0.06$ \\
\hline AT18-053 & 375 & $\mathrm{C} 2$ & South & 24 & $514.4 \pm 0.1$ & $507.4 \pm 0.1$ & $97.5 \pm 0.1$ & $2.82 \pm 0.11$ & $1.52 \pm 0.01$ & $1.37 \pm 0.05$ \\
\hline AT18-056 & 421 & $\mathrm{C} 2$ & South & 25 & $382.7 \pm 0.1$ & $374.7 \pm 0.1$ & $62.6 \pm 0.1$ & $2.82 \pm 0.11$ & $1.19 \pm 0.00$ & $1.07 \pm 0.04$ \\
\hline AT18-058 & 455 & $\mathrm{C} 2$ & South & 24 & $779.4 \pm 0.1$ & $769.3 \pm 0.1$ & $180.9 \pm 0.1$ & $2.82 \pm 0.11$ & $1.46 \pm 0.01$ & $1.27 \pm 0.05$ \\
\hline AT18-059 & 392 & $\mathrm{C} 2$ & South & 23 & $236.0 \pm 0.1$ & $233.1 \pm 0.1$ & $38.1 \pm 0.1$ & $2.82 \pm 0.11$ & $1.41 \pm 0.01$ & $1.29 \pm 0.05$ \\
\hline AT18-061 & 468 & $\mathrm{C} 2$ & South & 20 & $448.9 \pm 0.1$ & $430.0 \pm 0.1$ & $106.2 \pm 0.1$ & $2.82 \pm 0.11$ & $1.58 \pm 0.01$ & $1.38 \pm 0.05$ \\
\hline AT18-062 & 470 & $\mathrm{C} 2$ & South & 20 & $272.2 \pm 0.1 \mid$ & $270.3 \pm 0.1$ & $77.6 \pm 0.1$ & $2.82 \pm 0.11$ & $1.51 \pm 0.01$ & $1.27 \pm 0.05$ \\
\hline AT18-063 & 492 & $\mathrm{C} 2$ & Ridgetop & 5 & $91.3 \pm 0.1$ & $89.8 \pm 0.1$ & $7.7 \pm 0.1$ & $2.82 \pm 0.11$ & $1.37 \pm 0.02$ & $1.31 \pm 0.06$ \\
\hline AT18-106 & 455 & $\mathrm{C} 5$ & Ridgetop & 22 & $233.9 \pm 0.1$ & $231.4 \pm 0.1$ & $92.3 \pm 0.1$ & $2.82 \pm 0.11$ & $1.43 \pm 0.01$ & $1.08 \pm 0.04$ \\
\hline AT18-108 & 447 & $\mathrm{C} 5$ & South & 8 & $116.2 \pm 0.1$ & $115.0 \pm 0.1$ & $9.8 \pm 0.1$ & $2.82 \pm 0.11$ & $1.63 \pm 0.02$ & $1.57 \pm 0.07$ \\
\hline AT18-111 & 436 & $\mathrm{C} 5$ & South & 10 & $369.9 \pm 0.1$ & $366.0 \pm 0.1$ & $118.0 \pm 0.1$ & $2.82 \pm 0.11$ & $1.56 \pm 0.03$ & $1.29 \pm 0.06$ \\
\hline AT18-113 & 415 & $\mathrm{C} 5$ & South & 10 & $228.9 \pm 0.1$ & $226.6 \pm 0.1$ & $59.9 \pm 0.1$ & $2.82 \pm 0.11$ & $1.52 \pm 0.02$ & $1.30 \pm 0.06$ \\
\hline AT18-115 & 395 & $\mathrm{C} 5$ & South & 16 & $161.9 \pm 0.1$ & $159.3 \pm 0.1$ & $35.5 \pm 0.1$ & $2.82 \pm 0.11$ & $1.37 \pm 0.01$ & $1.19 \pm 0.05$ \\
\hline AT18-117 & 358 & $\mathrm{C} 5$ & South & 19 & $345.6 \pm 0.1$ & $341.5 \pm 0.1$ & $71.2 \pm 0.1$ & $2.82 \pm 0.11$ & $1.09 \pm 0.01$ & $0.94 \pm 0.04$ \\
\hline AT18-120 & 319 & $\mathrm{C} 5$ & South & 42 & $405.5 \pm 0.1$ & $400.9 \pm 0.1$ & $105.9 \pm 0.1$ & $2.82 \pm 0.11$ & $1.30 \pm 0.01$ & $1.09 \pm 0.04$ \\
\hline AT18-121 & 321 & $\mathrm{C} 5$ & North & 17 & $474.4 \pm 0.1$ & $470.7 \pm 0.1$ & $188.3 \pm 0.1$ & $2.82 \pm 0.11$ & $1.79 \pm 0.01$ & $1.43 \pm 0.06$ \\
\hline AT18-123 & 348 & $\mathrm{C} 5$ & North & 21 & $231.2 \pm 0.1$ & $228.5 \pm 0.1$ & $50.1 \pm 0.1$ & $2.82 \pm 0.11$ & $1.38 \pm 0.01$ & $1.20 \pm 0.05$ \\
\hline AT18-129 & 405 & $\mathrm{C} 5$ & North & 7 & $137.1 \pm 0.1$ & $135.1 \pm 0.1$ & $14.9 \pm 0.1$ & $2.82 \pm 0.11$ & $1.29 \pm 0.02$ & $1.21 \pm 0.05$ \\
\hline AT18-133 & 429 & $\mathrm{C} 5$ & North & 7 & $239.0 \pm 0.1$ & $236.1 \pm 0.1$ & $20.4 \pm 0.1$ & $2.82 \pm 0.11$ & $1.29 \pm 0.03$ & $1.23 \pm 0.06$ \\
\hline AT18-136 & 478 & $\mathrm{C} 5$ & North & 10 & $164.7 \pm 0.1$ & $163.2 \pm 0.1$ & $41.3 \pm 0.1$ & $2.82 \pm 0.11$ & $1.20 \pm 0.09$ & $1.00 \pm 0.11$ \\
\hline AT18-138 & 524 & C5 & Ridgetop & 4 & $138.1 \pm 0.1$ & $136.4 \pm 0.1$ & $16.7 \pm 0.1$ & $2.82 \pm 0.11$ & $1.38 \pm 0.02$ & $1.28 \pm 0.06$ \\
\hline
\end{tabular}

${ }^{a}$ DTM-derived.

${ }^{b}$ Soil pit depth below the surface as measured in the field.

${ }^{c}$ Field-state aliquot mass. 
${ }^{d}$ Oven-dried aliquot mass.

${ }^{e}$ Mass of rocks (> $2 \mathrm{~mm}$ ) in an aliquot sample.

${ }^{f}$ Bedrock density as measured by submerging bedrock and unweathered saprolite samples from catchment C5 under water $(n=28)$.

${ }^{g}$ Dry bulk density. Uncertainties include the $1 \sigma$ uncertainty in volume as obtained from the tests performed on the artificial pit and the accuracy of the balance used.

${ }^{h}$ Dry soil density. Uncertainties include the $1 \sigma$ uncertainty in volume as obtained from the tests performed on the artificial pit and the accuracy of the balance used.

\section{S4 Regular freeware workflow as applied in this study}

To facilitate the application of density measurements following the workflows presented here, we give a detailed protocol of the freeware computing steps involved below.

Mesh generation using Regard3D (ver. 1.0.0)

1) Upload the pictures (Add Picture Set $\rightarrow$ Add files). Create two picture sets per model, one set containing photographs from the pre-dug surface and one set containing the pictures taken from the excavated pit. Name the picture sets accordingly.

2) Compute matches. If needed, Image correlations parameters can be adjusted. We found that increasing the keypoint sensitivity can improve the final mesh significantly. Regularly, all default parameters can be used.

3) Create the sparse cloud (Triangulation). If the Triangulation is not successful, the Triangulation parameters can be changed to Old incremental Structure from Motion, where an initial image pair has to be selected. Regularly, all default parameters can be used.

4) Densify the point cloud (Create dense pointcloud). If image contrasts are high and the photographs generally bright and colourful, as it should be the case for the majority of photographs taken in the field, all default parameters can be used. In some cases, we changed the Minimum image number to 2. If this does not work, the Densification method can be changed to Multi-View Environment $(M V E)$. Further improvements using this method can be achieved by decreasing the Scale and increasing the Filter width at the expense of additional computing time. For the reconstruction of the artificial pit we used for testing purposes, we often applied a Scale of 3 and a Filter width of 5.

5) Create the mesh. We used the Poisson surface reconstruction to create the surfaces. For the alignment of the pre-dug and pit surfaces it can be useful to create Textures that may allow for a more accurate tie point assignment (Colorization method $\rightarrow$ Textures). We found that Textures will be most accurately placed on the surfaces if Photometric outlier removal under Texturization parameters is set to Gauss damping, with Geometric visibility test, Global seam levelling, and Local seam levelling are being activated. All other parameters can be left as default. 
6) Save the surfaces as OBJ file using Export surface. If Textures have been created, the surface should be saved into a new folder, separated from other exported surfaces.

\section{$\underline{\text { Scaling, surface alignment and merging in CloudCompare (ver. 2.11) }}$}

7) Import the surfaces (Open or Drag and Drop).

8) Click on the respective meshes and check the Visible box in the Properties window, uncheck the Normals box. Now the textured meshes should be displayed.

9) It might be necessary to roughly clip the reconstructed pit in order to facilitate further processing. In the Main tools toolbar, chose the Segment tool. Cut out the pit.

10) Zoom on a scale within a scene (i.e. a scale bar or an object with known measures). In the Main tools toolbar, chose the Point picking tool. Use the Select 2 points and display segment information function of the tool to trace the length of the scale. Place two points on the scale with a known distance from each other (e.g. on the 1-cm-mark and on the 4-cm-mark of a folding rule), as visible from the model's texture.

11) Divide the measured distance by the true distance to obtain the scaling factor (e.g. the true length between the 1-cm-mark and 4-cm-mark is $3.00 \mathrm{~cm}$, and the distance measured is 1.15 length units (lu), then the scaling factor would be $3.00 \mathrm{~cm} / 1.15 \mathrm{lu}=2.61 \mathrm{~cm} \mathrm{lu}^{-1}$ ).

12) Navigate to Edit $\rightarrow$ Multiply/Scale and insert the scaling factor. Make sure that the options Same scale for all dimensions and Rescale Global shift are both checked.

13) The scaling of the pit mesh might have caused a large contrast in size between pit and pre-dug surface meshes. A rough scaling can thus also be applied to the pre-dug surface mesh in order to facilitate the alignment procedure. Alternatively, the mesh can be automatically scaled using the Match scales tool (Tools $\rightarrow$ Registration).

14) If necessary, roughly clip the pre-dug surface mesh (see \#9).

15) In the $D B$ Tree, select both the pre-dug surface and the pit mesh. In the Main tools toolbar, select Align (point pairs picking). Chose the scaled pit mesh as Reference.

16) Mark four or more point pairs in both meshes to align the models. By clicking on align, a preview of the alignment, including a display of alignment errors, can be obtained. The final alignment can be approved by clicking on the green tick.

17) Clip the aligned meshes to their outer rims, i.e. to the extent where the pre-dug surface has been modified by digging. To facilitate this working step, the meshes can be colourised in different colours to enhance surface contrasts (Edit $\rightarrow$ Colors $\rightarrow$ Set unique). Textures can be hidden by removing the tick at Materials/textures in Mesh (Mesh Properties). Alternatively, clipping can be executed after step \#18. 
18) Merge the two meshes by using the Merge multiple clouds tool (Main tools toolbar). Make sure that both meshes are selected. A new Merged mesh will appear in the DB Tree.

19) Export the merged mesh by selecting it in the DB Tree and clicking on Save in the Main tools toolbar. In this work, the files were saved as PLY for convenience.

\section{Building the watertight mesh using Microsoft 3D Builder (ver. 18.0.1931.0)}

20) Import the mesh in Microsoft 3D Builder (Load object or Drag and Drop).

21) Chose the File's units and import the model (Import model). A window should appear with a message notifying the user that One or more objects are invalidly defined. Click here to repair. Click on the message, and the program will automatically bridge the voids between the two surfaces.

22) Export the watertight mesh (Menu $\rightarrow$ Save as). The volume can now be obtained from various programs, e.g. CloudCompare or Meshlab (ver. 2016.12).

23) In those cases where the two surfaces did not sufficiently overlap after merging, the bridging as implemented in the Microsoft 3D Builder might fail (creating two single watertight meshes instead of one). To resolve this problem, the bridging must be performed manually by establishing a connection between the two meshes. We used netfabb Basic (ver. 7.4.0) to achieve this. The merged mesh can be imported into the program (Project $\rightarrow$ Add Part or Drag and Drop). Under Extras, chose Repair part. Zoom to the outer rim of the merged mesh, where the two surfaces should be about to converge. Use the Add Triangles tool (Mesh Edit) and click on the outer edge of the predug surface; then click on the closest outer edge of the pit surface. After the bridge has been created, execute Apply Repair. In the Context Area, the modified mesh can be exported by right-clicking on the mesh and choosing Export Part. Once this step is performed, continue with step \#20. 